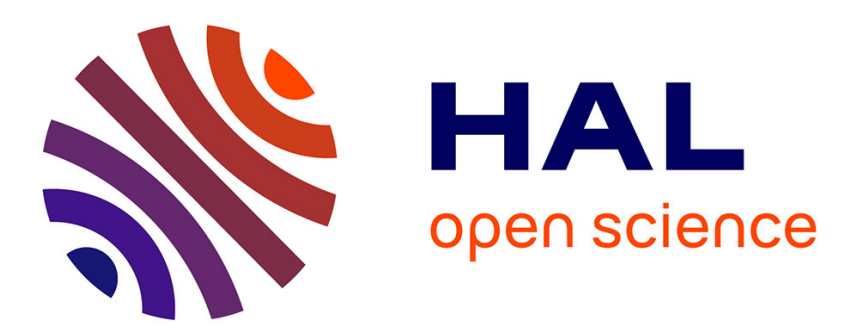

\title{
Voltage/frequency proportional control of stick-slip micropositioning systems.
}

Micky Rakotondrabe, Yassine Haddab, Philippe Lutz

\section{To cite this version:}

Micky Rakotondrabe, Yassine Haddab, Philippe Lutz. Voltage/frequency proportional control of stick-slip micropositioning systems.. IEEE Transactions on Control Systems Technology, 2008, 16 (6), pp.1316-1322. 10.1109/TCST.2008.917232 . hal-00340191

\section{HAL Id: hal-00340191 https://hal.science/hal-00340191}

Submitted on 20 Nov 2008

HAL is a multi-disciplinary open access archive for the deposit and dissemination of scientific research documents, whether they are published or not. The documents may come from teaching and research institutions in France or abroad, or from public or private research centers.
L'archive ouverte pluridisciplinaire HAL, est destinée au dépôt et à la diffusion de documents scientifiques de niveau recherche, publiés ou non, émanant des établissements d'enseignement et de recherche français ou étrangers, des laboratoires publics ou privés. 


\title{
Voltage/frequency proportional control of stick-slip micropositioning systems
}

\author{
Micky Rakotondrabe, member IEEE, Yassine Haddab and Philippe Lutz, member IEEE
}

\begin{abstract}
A new control type for stick-slip micropositioning systems is proposed in this paper: the voltage/frequency (U/f) proportional control. It gives more precise results relatively to the classical control algorithm. It is also an assembling of two classical controllers: the sign and the classical proportional controllers. A high stroke model of a stick-slip micropositioning system is first given. Then, we will theoretically analyze the performances of the closed loop process with the U/f controller. Finally, we will give some experimental results obtained with different values of the proportional gains.
\end{abstract}

Index Terms-Stick-slip, micropositioning system, control, U/f proportional control, Lyapunov stability.

\section{INTRODUCTION}

To assemble and to process microproducts, especially MEMS (MicroElectroMechanical Systems), the use of conventional assembly systems leads to major difficulties because of the scale effects (example: prevalence of the adhesion forces relative to the weight [1]) and the complexity of the physics of the microworld. In order to take into account these scale effects, a complete study of the actuators, the robots, the sensors and the production methods must be done. In addition, they must offer high resolution and high accuracy. For example, the articulations are replaced by deformable materials in microactuators while visual feedback is used more and more as a sensor. Concerning the microrobots, new motion principles have been developped in order to obtain high stroke and high resolution: the inch-worm, the stick-slip and the impact-drive principles. These are stepping motions. Due to their simplicity, rapidity, low cost and the possibility of batch fabrication, the stick-slip one is the most used: as in references [2] [3] [4] [5] [6] [7].

Stick-slip actuators are generally based on piezoelectric materials. Two modes of motion can be obtained with a micropositioner using stick-slip actuators [8]: the stepping mode and the scanning mode. The stepping mode consists of applying a sawtooth voltage to the micropositioner and letting it move step by step, in high range and with a high velocity on the workspace (Fig. 1-a, b and c). The resolution in this mode is limited to one step. When the difference between the target position and the present postion becomes less than the value of a step, the legs (piezoelectric actuators) are slowly

\footnotetext{
Laboratoire d'Automatique de Besançon

LAB CNRS UMR6596

ENSMM - Université de Franche-Comté

24 , rue Alain Savary

25000 Besançon - France

\{mrakoton, yhaddab, plutz\} dens $2 \mathrm{~m}$. fr

Paper type: brief paper. Initial submission: September 2006. Accepted: July
} 2007 bent until the final position is reached (Fig. 1-d). This is the scanning mode and the obtained resolution can be very high. It is possible to have a similar resolution in the stepping mode by diminishing the amplitude $U$ of the sawtooth but the vibrations which occur in each step [9] may influence the performances.

There are two modes of control for stick-slip micropositioners: control in stepping mode for high stroke positioning and control in scanning mode for fine positioning. Each of them may be either an open-loop or a closed-loop structure. As the fine positioning control may be closed-loop with standard controllers ( $P I D$, optimal controllers, robust controllers etc.), this paper only deals with the high stroke positioning control.

The first section is a brief presentation of existing control methods. Then, we will present the high stroke characteristics and model of our stick-slip micropositioning system for experiments. After that, we will detail a new type of control: the voltage/frequency proportional control. Finally, we will present the experimental and simulation results.

\section{EXISTING METHODS}

Open-loop control based on step counting as in stepper motors are not applicable for stick-slip micropositioning systems because:

- in general, the steps are never identical along a displacement due to the nonlinearities phenomena,

- the step is very small relatively to the distance so that a small arror in counting gives a high statical error,

- the step errors $\left(s_{\text {tep }}\right.$ errors $=$ step $\left._{\text {estimated }}-s_{\text {tep }} p_{\text {real }}\right)$ accumulate along the motion unlike in stepper motors,

- the external disturbances (adhesion forces, thermal effect, vibrations of the workspace etc.) influence indeniably the accuracy.

Thus, closed loop controllers were introduced. First, there is the basic algorithm. We decided to introduce the term "basic" because it uses the basic instructions in programming. The algorithm except for a sign is as follow:

$$
\begin{aligned}
& \text { While }\left|x_{c}-x\right| \geq \text { step Do } \\
& \quad \text { apply one step } \\
& \text { EndWhile }
\end{aligned}
$$

where $x_{c}$ is the target position and $x$ is the current position. When the accuracy of the sensor is worse than the value of a step, a slight alteration must be made to the precedent algorithm in order to avoid permanent oscillations:

$$
\begin{aligned}
& \text { While }\left|x_{c}-x\right| \geq n \times \text { step Do } \\
& \quad \text { apply } n \times \text { step } \\
& \text { EndWhile }
\end{aligned}
$$




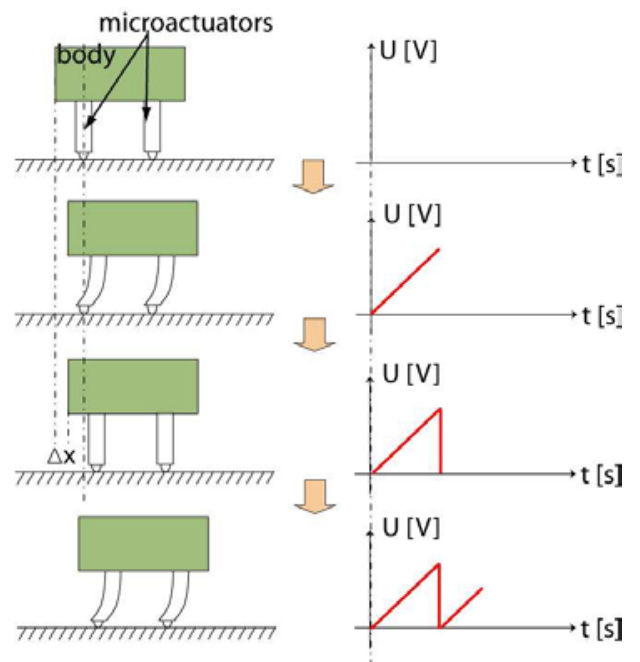

Fig. 1. Stick-slip principle. a, b and c: stepping mode. d: scanning mode.

where $n \times$ step gives the limit of the sensor accuracy.

Another method based on the hybrid modeling of the stickslip systems was proposed in [10](Fig. 2). It consists of approximating the hybrid model into a continuous one (called dehybridization) and applying a continuous controller $(P D)$. The principal advantage of this method is the possibility to reach the target position without using two separate control modes (stepping and scanning). However, the dehybridization necessitates a hybrid controller in series with the hybrid plant which make the whole algorithm more complex than the classical one.

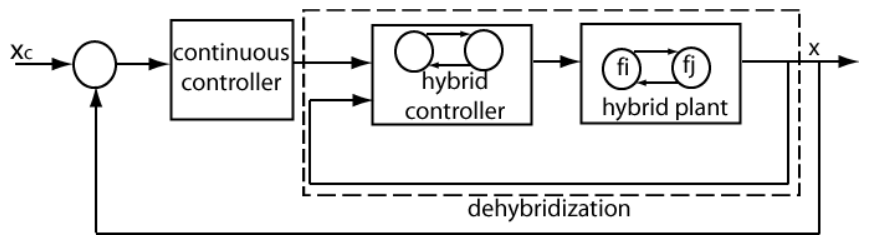

Fig. 2. Control of stick-slip systems based on the dehybridization method [10].

Finally, a numerical frequency proportional controller was proposed in [11]. It combines the two modes of motion (stepping mode and scanning mode) with a very simple way. The position error is converted into a clock signal whose frequency is proportional to the error. According to the error's sign, the output signal of a counter is increment or decrement. A DAC converts the numeric signal into a sawtooth signal with a frequency proportional to the error and a constant amplitude. When the position tends towards the target position, the frequency tends towards zero. That is equivalent to a scanning mode control. However, all along the displacement, the resolution is constant.

\section{CHARACTERISTICS AND MODELLING OF THE MICROPOSITIONING SYSTEM}

Fig. 3 shows the stick-slip micropositioning system [12] used for experiments in this paper. It has two degrees of freedom (2DoF: linear and angular) but our test will only be performed in the linear motion. The maximum step size of the micropositioning system is about $200 \mathrm{~nm}$ and the speed can reach $2 \mathrm{~mm} / \mathrm{s}$. These are obtained with a sawtooth input voltage of $\pm 150 \mathrm{~V}$ amplitude and $10 \mathrm{kHz}$ frequency.

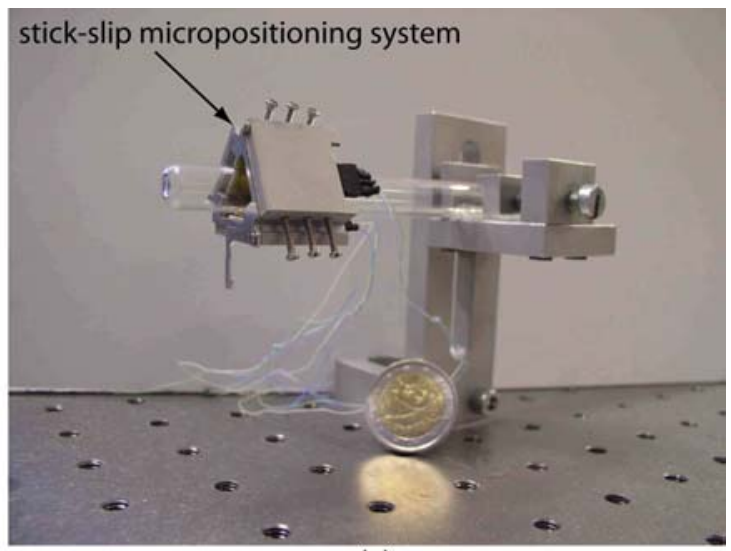

(a)

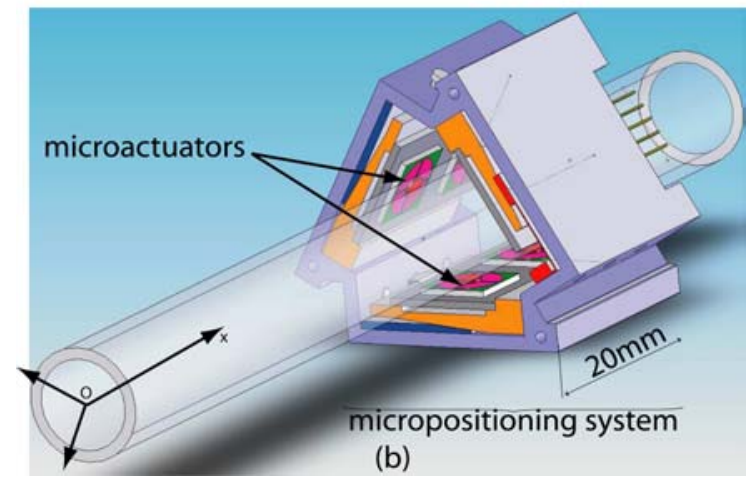

Fig. 3. The stick-slip micropositioning system.

When applying a continuous input voltage $U$, the micropositioning system works in scanning mode. Hypothesizing that the scanning displacement $x_{s c}$ is dynamically linear relative to the voltage, we can write the following function:

$$
x_{s c}(s)=G_{s c}(s) \cdot U(s)
$$

Where $G_{s c}$ is a linear transfer function and $s$ represents the Laplace variable. When the voltage is set to zero, the resulting step $x_{\text {step }}$ is smaller than the corresponding amplitude $x_{s c}^{U}$ so that (Fig. 4-a):

$$
x_{\text {step }}=x_{s c}^{U}-\Delta_{b a c k}
$$

Hypothesizing that the backlash $\Delta_{b a c k}$ is dynamically linear relatively to the amplitude $U$, the step can be written as follow:

$$
x_{\text {step }}(s)=G_{\text {step }}(s) \cdot U(s)
$$

Where $G_{\text {step }}$ is a linear transfer function. When the sequence is repeated (stepping mode) with a frequency $f=1 / T$, the dynamic of the continous part $G_{\text {step }}$ is not visible and the micropositioning system works with a quasi-static manner. Thus, the step can be approximated by:

$$
x_{\text {step }}=\alpha \cdot U
$$


where $\alpha>0$ is the statical gain of $G_{\text {step }}$.

From Fig. 4-b and the (Equ 6), we infer the bilinearity of the speed:

$$
v=\dot{x}=\alpha \cdot U \cdot f
$$

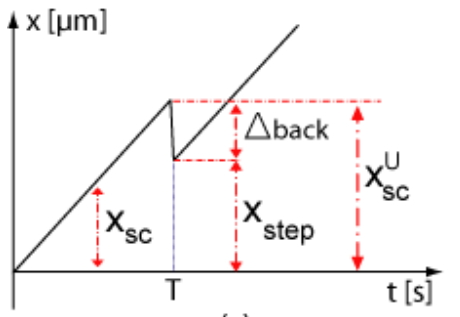

(a)

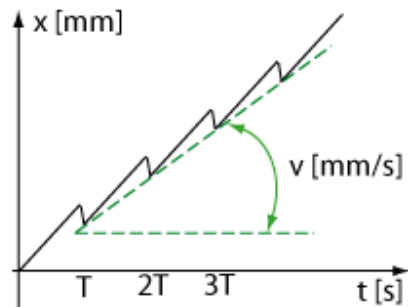

(b)
Fig. 4. a: motion of a stick-slip system. b: speed approximation.

When the amplitude $U$ is below a certain value $U_{0}$, the torque is not sufficient and the micropositioning system moves back and forth in scanning mode. An offset is then introduced to the (Equ 6) and the final model becomes:

$$
\left\{\begin{array}{ccc}
v=0 & \text { if } & |U| \leq U_{0} \\
v=\alpha \cdot f \cdot\left(U-\operatorname{sgn}(U) \cdot U_{0}\right) & \text { if } & |U|>U_{0}
\end{array}\right.
$$

Fig. 5 summarizes the speed performances of the micropositioning system. Until $f=10 \mathrm{kHz}$, the speed is approximately linear versus $f$ (Fig. 5-a). Above this frequency value, there is saturation and fluctuation. The identified parameters are: $\alpha=15.65 \times 10^{-7} \mathrm{~mm} / \mathrm{V}$ and $U_{0}=35 \mathrm{~V}$ (Fig. 5-b).

\section{U/F PROPORTIONAL CONTROL}

\section{A. Principle scheme}

The principe scheme of the U/f proportional control is shown in Fig. 6. The voltage saturation and the frequency saturation allow overvoltages to be avoided and limit the micropositioning system work inside the linear frequential zone. The proportional gains $K_{U}>0$ and $K_{f}>0$ are to be adjusted like in a classical proportional controller.

\section{B. Equations and analysis}

In this section, we express the speed according to the values of $K_{U}$ and $K_{f}$. Let $U_{s}$ and $f_{s}$ indicate the saturations respectively used for the voltage and for the frequency.

a) - if $K_{U} \cdot\left|x_{c}-x\right|>U_{s}$ and $K_{f} \cdot\left|x_{c}-x\right|>f_{s}$ From Fig. 6 and the bi-afine expression in (Equ 6), we obtain:

$$
\dot{x}=\alpha \cdot f_{s} \cdot\left(U_{s}-U_{0}\right) \cdot \operatorname{sgn}\left(x_{c}-x\right)
$$

This case is equivalent to a sign control (Fig. 7). There are oscillations in sign control. The frequency and the amplitude of these oscillations depend on the response time $T_{r}$ of the process, on the updating time $T_{s}$ of the controller and on the frequency saturation $f_{s}$. It is in this way that realtime feedback systems give their best performances.

b) - if $U_{0}>K_{U} \cdot\left|x_{c}-x\right| \quad \forall \quad f=K_{f} \cdot\left|x_{c}-x\right| \quad$ we have:

$$
\dot{x}=0
$$
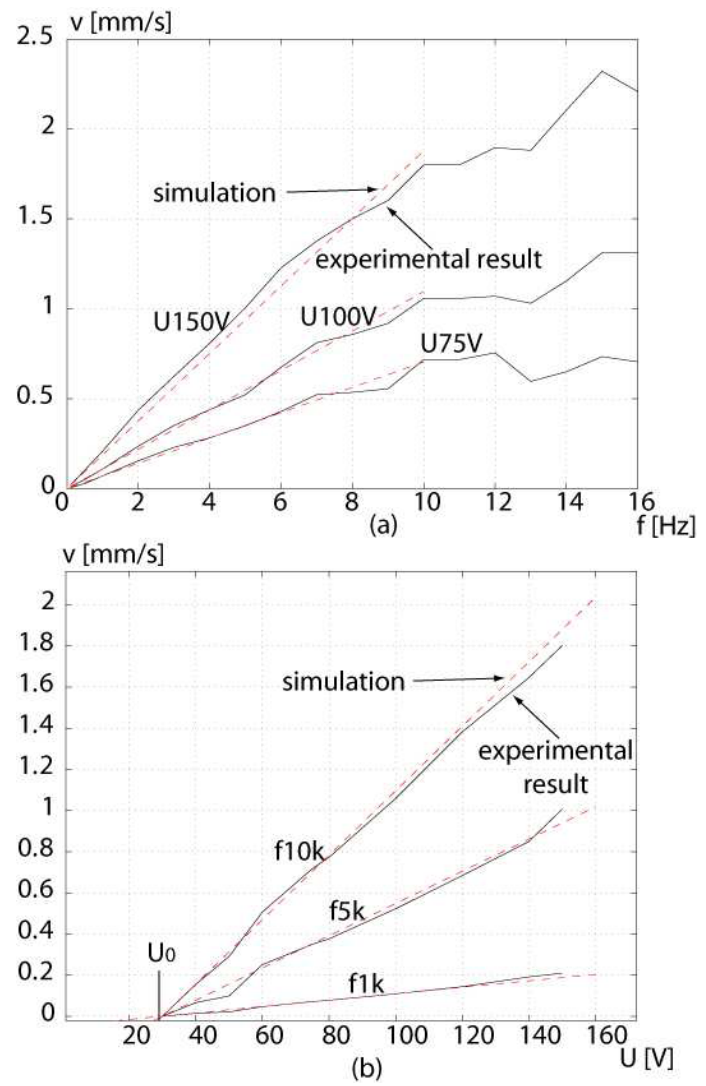

Fig. 5. Speed performances of the micropositioning system (experimental results in solid plots and simulation of (Equ 8) in dashed-plots). a: speed versus the frequency $f$. b: speed versus the amplitude $U$.

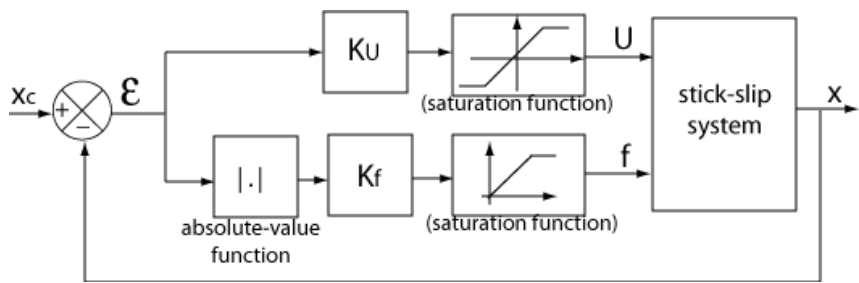

Fig. 6. Principle scheme of the U/f proportional control.

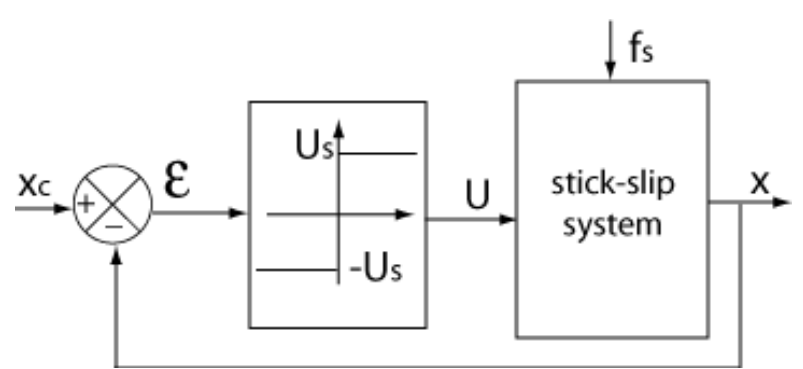

Fig. 7. Sign control.

c) - if $U_{s} \geq K_{U} \cdot\left|x_{c}-x\right| \geq U_{0} \quad$ and $\quad K_{f} \cdot\left|x_{c}-x\right|>f_{s}$ Here, the closed loop process is equivalent to a voltage proportional control (Fig. 8), also known as proportional control in the control theory. 


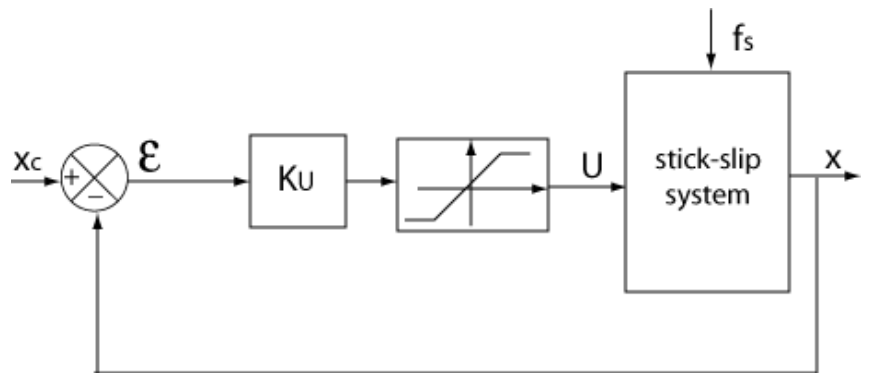

Fig. 8. Voltage proportional control.

The equation of the closed loop is:

$$
\dot{x}=\alpha \cdot f_{s} \cdot\left(K_{U} \cdot\left(x_{c}-x\right)-\operatorname{sgn}\left(x_{c}-x\right) \cdot U_{0}\right)
$$

To simplify, but without loss of generality, let us take a positive target position $x_{c}$ and an initial value $x(t=0)$ equal to zero, we obtain the following Laplace transformation:

$$
X=\frac{1}{1+\frac{1}{\alpha \cdot f_{s} \cdot K_{U}} \cdot s} \cdot X_{c}-\frac{\frac{1}{K_{U}}}{1+\frac{1}{\alpha \cdot f_{s} \cdot K_{U}} \cdot s} \cdot U_{0}
$$

The (Equ 12) means that the closed loop process is a first order dynamic system with a statical gain equal to unity and a disturbance $U_{0}$. We can infer that, the greater $K_{U}$ is, the more accurate a voltage proportional control of a stick-slip system is. The voltage proportional control always gives stability because of its order: first order.

d) - if $K_{U} \cdot\left|x_{c}-x\right|>U_{s}$ and $f_{s} \geq K_{f} \cdot\left|x_{c}-x\right|$ This case is equivalent to a frequency proportional control (Fig. 9).

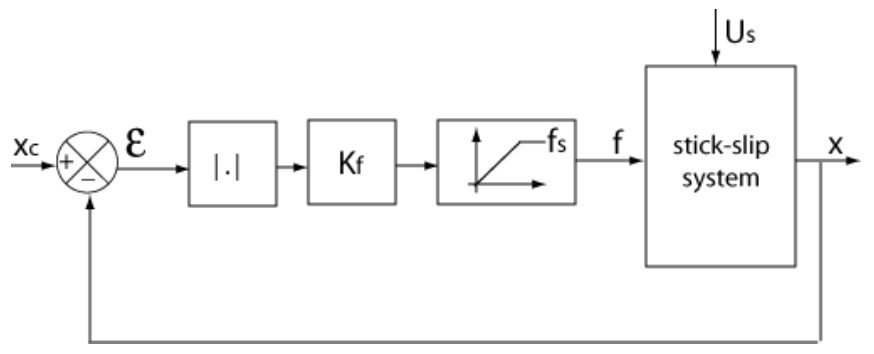

Fig. 9. Frequency proportional control.

The following equation is easily obtained:

$$
\dot{x}=\alpha \cdot K_{f} \cdot\left|x_{c}-x\right| \cdot\left(U_{s}-U_{0}\right) \cdot \operatorname{sgn}\left(x_{c}-x\right)
$$

The expression (Equ 13) is equivalent to the following transfer function:

$$
\frac{X}{X_{c}}=\frac{1}{1+\frac{1}{\alpha \cdot K_{f} \cdot\left(U_{s}-U_{0}\right)} \cdot s}
$$

The expression (Equ 14) means that in this case, the closed loop process is a first order system with a statical gain equal to unity. Stability is always ensured.

e) - if $U_{s} \geq K_{U} \cdot\left|x_{c}-x\right| \geq U_{0} \quad$ and $f_{s} \geq K_{f} \cdot \mid x_{c}-x$ The frequency and the voltage are both proportional to the error $\varepsilon$. From Fig. 6 and the formula (Equ 8), we have the following expression:

$$
\dot{x}=\alpha \cdot K_{f} \cdot\left|x_{c}-x\right| \cdot\left(K_{U} \cdot\left(x_{c}-x\right)-\operatorname{sgn}\left(x_{c}-x\right) \cdot U_{0}\right)
$$

The expression (Equ 15) is equivalent to:

$$
\begin{aligned}
& \frac{d x}{d t}=\left(\alpha \cdot K_{f} \cdot U_{0}-\alpha \cdot K_{f} \cdot K_{U} \cdot\left|x_{c}-x\right|\right) \cdot x \\
& +\left(-\alpha \cdot K_{f} \cdot U_{0}+\alpha \cdot K_{f} \cdot K_{U} \cdot\left|x_{c}-x\right|\right) \cdot x_{c}
\end{aligned}
$$

which is in the form of:

$$
\frac{d x}{d t}=A\left(x_{c}, x\right) \cdot x+B\left(x_{c}, x\right) \cdot x_{c}
$$

Here, the closed loop system has a first order pseudo-linear behavior.

\section{Stability}

According to the values of $K_{U}, K_{f}$ and the error $\left(x_{c}-x\right)$, all the above cases may appear during a displacement. Let us suppose that $x_{c}=0$ and $x(t=0)>0$ for the stability analysis. Let us divide the displacement (Fig. 10) into two phases :

- phase-1; the error $\left(x_{c}-x\right)$ is initially high so that the voltage $U$ and the frequency $f$ are both in their state of saturation (case-a). The speed is constant.

- phase-2; the error becomes smaller and the speed is not yet constant (equivalent to the rest of the cases).

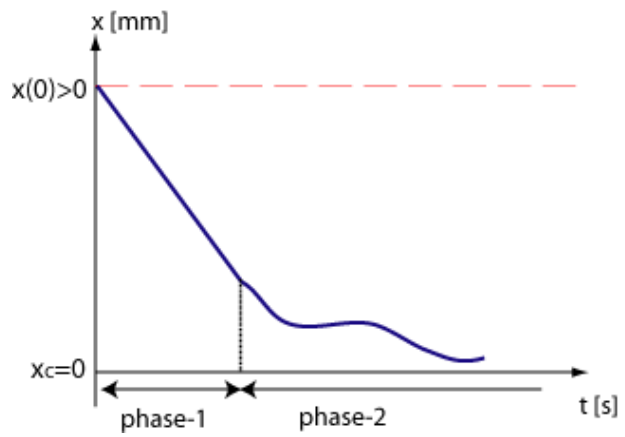

Fig. 10. The displacement may be divided into two phases.

As the equations are quasi-static, ie. there is no acceleration, one case does not influence the succeeding case. So, phase2 may be studied independantly of phase-1. In phase-2, two sub-phases occur:

- phase-2.1; either the frequency is saturated but not the voltage (case-c) or the voltage is saturated but not the frequency (case-d),

- phase-2.2; both the frequency and the voltage are not saturated (case-e).

Once again, phase-2.1 does not influence phase-2.2. As the two possible cases inside phase-2.1 are with 1st order linear behaviors, the displacement from the initial position to the present position has not been yet overshot. On the other hand, when the voltage becomes smaller than $U_{0}$ (case-b), the stick-slip micropositioning system stops independantly of the precedent phases. Thus, the stability analysis of the 
micropositioning system may be done only with phase- 2.2 (case-e). For that, we use the direct method of Lyapunov.

A dynamic system $\frac{d x}{d t}=f(x, e, t)$, where $e$ is the input vector, is Lyapunov stable if there exists a Lyapunov function $V(x)$ such as:

$$
\begin{gathered}
V(x=0)=0 \\
V(x)>0 \quad \forall \quad x \neq 0 \\
\frac{d V(x)}{d t} \leq 0 \quad \forall \quad x \neq 0
\end{gathered}
$$

From (Equ 16) and the conditions $x(t=0)>0$ and $x_{c}=0$, we obtain:

$$
\frac{d x}{d t}=-\alpha \cdot K_{f} \cdot x \cdot\left(K_{U} \cdot x-U_{0}\right)
$$

We use the following quadratical functional $V(x)$ :

$$
V(x)=\gamma \cdot x^{2}
$$

where $\gamma$ is a positive constant. The conditions (Equ 18) and (Equ 19) are filled.

From (Equ 21) and (Equ 22), we have:

$$
\frac{d V(x)}{d t}=-2 \cdot \gamma \cdot \alpha \cdot K_{f} \cdot x^{2} \cdot\left(K_{U} \cdot x-U_{0}\right)
$$

As $\left(K_{U} \cdot x-U_{0}\right)>0$, the condition (Equ 20) is filled and all the trajectory is asymptotically (Lyapunov) stable. When $\left(K_{U} \cdot x-U_{0}\right)<0$, the micropositioning system stops, the stability is obvious and the statical error is given.

\section{EXPERIMENTS}

The experimental setup is composed of a computer, an amplifier, the micropositioning system and a laser sensor (resolution $0.5 \mathrm{~nm}$, accuracy $10 \mu \mathrm{m}$ ). The computer has no realtime operating system (Windows-XP) and we use LabView software for implementation of the U/f controller.

The choice of $K_{U}$ is a compromise. If $K_{U}$ is very low, the statical error is high. If $K_{U}$ is very high, there is a risk of oscillations (case-a) when the refreshing time $T_{s}$ is not negligible. For all the experiments, the target point $x_{c}=$ $10 \mathrm{~mm}$ and the initial point $x(0)=0 \mathrm{~mm}$.

The first experiment consists of giving high values to $K_{u}$ and $K_{f}$. They have been chosen so that phase- 2 never occurs. This corresponds to the case-a. Fig. 11 gives the experimental and the simulation curves. Due to the non real-time of the operating system, the experiment provides oscillations (Fig. 11in solid plot).

Secondly, we use a low $K_{U}$ and a high $K_{f}$. The frequency always remains saturated while the voltage becomes non saturated when $x_{c}-x$ is inferior to a certain value $x_{U S}$ (Fig. 12-a) such as $x_{U S}=U_{s} / K_{U}$. After that, the behavior is equivalent to (Equ 12) of the case-c, ie. a voltage proportional control. As shown in the figure, there is a statical error. Its value is equal to $\varepsilon_{\text {stat }}=U_{0} / K_{U}$.

Then, we experiment with a high $K_{U}$ but a low $K_{f}$ (Fig. 12b). The position error at which we leave the saturation is obtained at $\left(x_{c}-x\right)=x_{f S}=f_{s} / K_{f}$. From this point, the closed loop system has the frequential proportional behavior and there is no statical error (case-d).

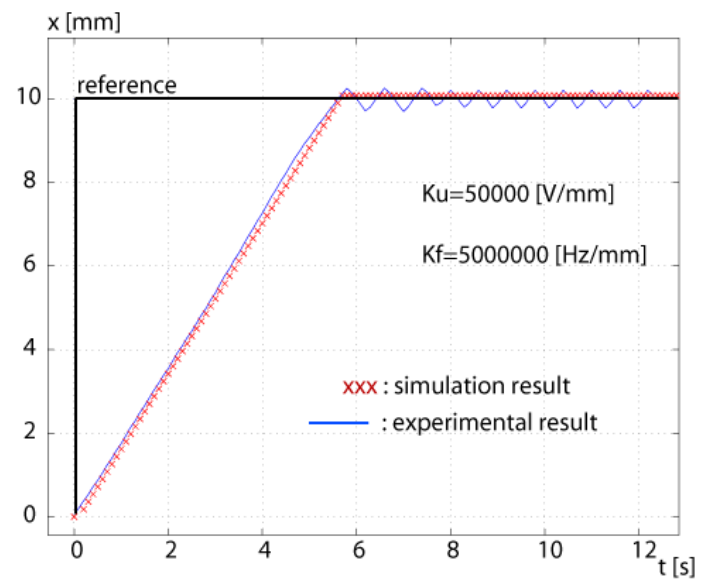

Fig. 11. High values of $K_{U}$ and $K_{f}$ : case-a.
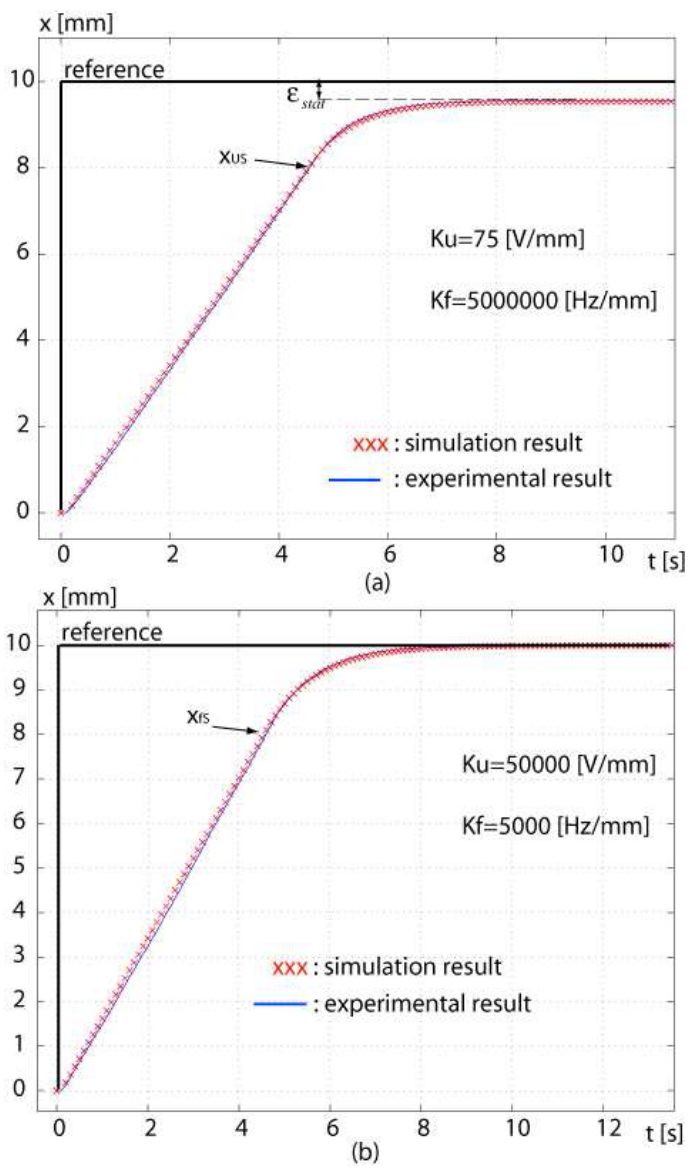

Fig. 12. a: low $K_{U}$ and high $K_{f}$. b: high $K_{U}$ and low $K_{f}$.

Finally, we test values of $K_{U}$ and $K_{f}$ which appear to be reasonable (Fig. 13). First, the frequency leaves the saturation at $x_{f s}=f_{s} / K_{f}$ while the voltage remains saturated. When arriving at $x_{U S}=U_{s} / K_{U}$, the voltage is no longer in a state of saturation. According to the values of $K_{U}$ and $K_{f}$, the inverse circumstance may occur. The statical error is choosen so that $\varepsilon_{\text {stat }}=U_{0} / K_{U}$. 


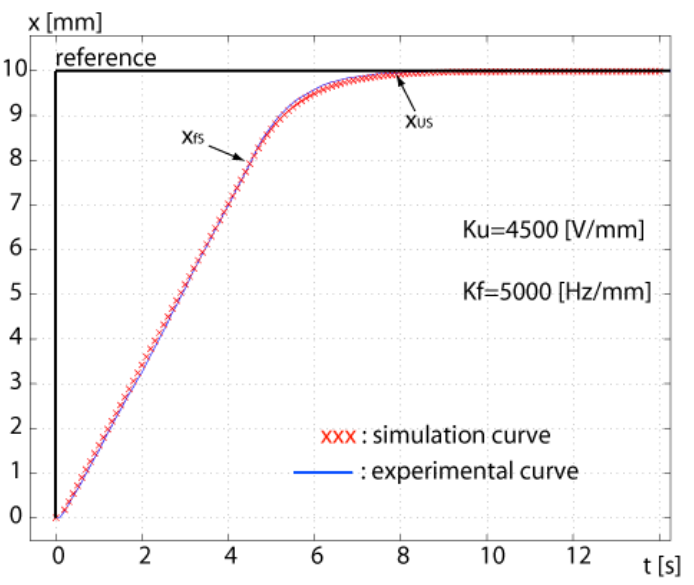

Fig. 13. Decent values of $K_{U}$ and $K_{f}$.

\section{DISCUSSION}

On the one hand, the U/f proportional controller encompasses three existing controllers: the sign controller (see casea), the classical proportional controller (see case-c) and the frequency proportional controller (see case-d) which has been used for stick-slip micropositioning systems. On the other hand, in comparison with the basic algorithm of (Equ 1) (respectively (Equ 2)), the U/f proportional controller gives a better resolution. The resolution obtained with this algorithm is one step (respectively $n \times$ step) while the one obtained with the U/f is better than one step, within the limits of the sensor accuracy. In fact, the diminution of the applied voltage $U=K_{U} \cdot \varepsilon$ reduces the value of a step. In addition, the diminution of the frequency $f=K_{f}$. $\varepsilon$ reduces the number of steps, and consequently the vibrations.

A test has been performed with an interferometer sensor (resolution $=1.24 \mathrm{~nm}$ ) in order to compare the performances obtained with the basic algotrithm (Equ 1) and the U/f proportionnal controller. The setup used is composed of materials with important values of $T_{s}$ and $T_{r}$ : the interferometer datagate and a Labview software inside Windows-XP. Thus, we use a low frequency $(1 \mathrm{~Hz})$ for the basic algorithm in order to avoid oscillations.

Fig. 14 shows the results with a setpoint (reference) of $10 \mu \mathrm{m}$. The zoom (Fig. 14-b) indicates that the statical error (accuracy) is about $130 \mathrm{~nm}$. As we can see, the resolution before the stop is the value of half of a step as opposed to a step because the applied voltage is a peak-to-peak signal $( \pm 150 \mathrm{~V})$, then when stopping it, the only half is cut out $(+150 \mathrm{~V} \rightarrow 0 \mathrm{~V})$.

Fig. 15 gives the results obtained with the U/f controller when applying a setpoint of $10 \mu m$. The zoom (Fig. 15-b) indicates that the frequency and the step amplitude become increasingly small. The medium statical error also becomes increasingly small. However, the backlash $\Delta$ back becomes increasingly significant. That is due to the diminution of the amplitude $U$ so that there will not be enough torque (acceleration) to move the micropositioning system during the slip phase. If we choose a higher value of the gain $K_{U}$, the statical error decreases. When $K_{U}$ is very high, the statical
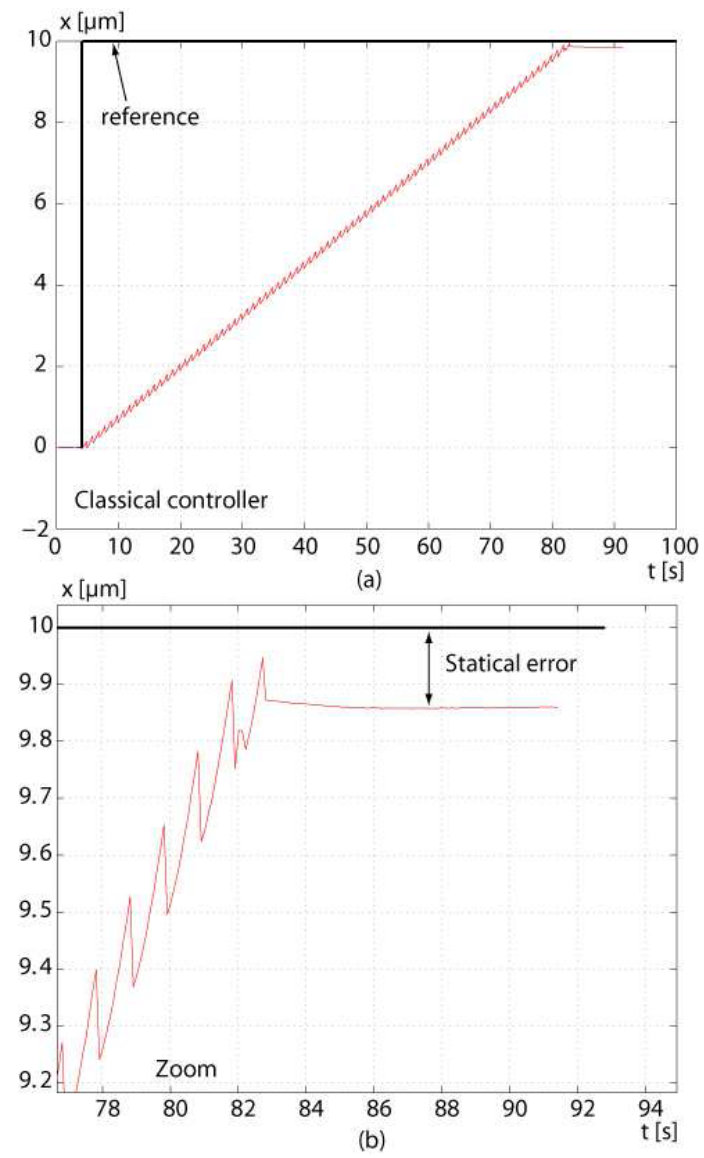

Fig. 14. Classical controller using an interferometer sensor. a: high stroke motion. b: zoom of the final position.

error tends towards zero. However, according to the response time $T_{s}$ and the refreshing time $T_{r}$, this case may lead to oscillations. Thus, using a faster controller setup would enable the implementation of a higher value of $K_{U}$.

\section{CONCLUSION}

A new controller type for high stroke displacement of stick-slip micropositioning systems was proposed: the voltage/frequency proportional controller. Like a classical proportional controller, it gives a frequency $f$ and an amplitude $U$ which are proportional to the error. To analyze the performances given by such a controller, we firstly modelled the high stroke displacement of the micropositioning system. Then, we analysed the stability according to the value of the proportional gains $K_{U}$ and $K_{f}$. Finally, we presented different cases through experiments and simulations. On the one hand, the U/f proportional control is a globalization of three existing controllers: the sign controller, the classical proportional controllers and the frequency proportional controller. On the other hand, the U/f proportional control gives better resolution than the basic algorithm. Finally, in the case of the frequential controller, the statical error tends towards zero and the accuracy is then very high. 

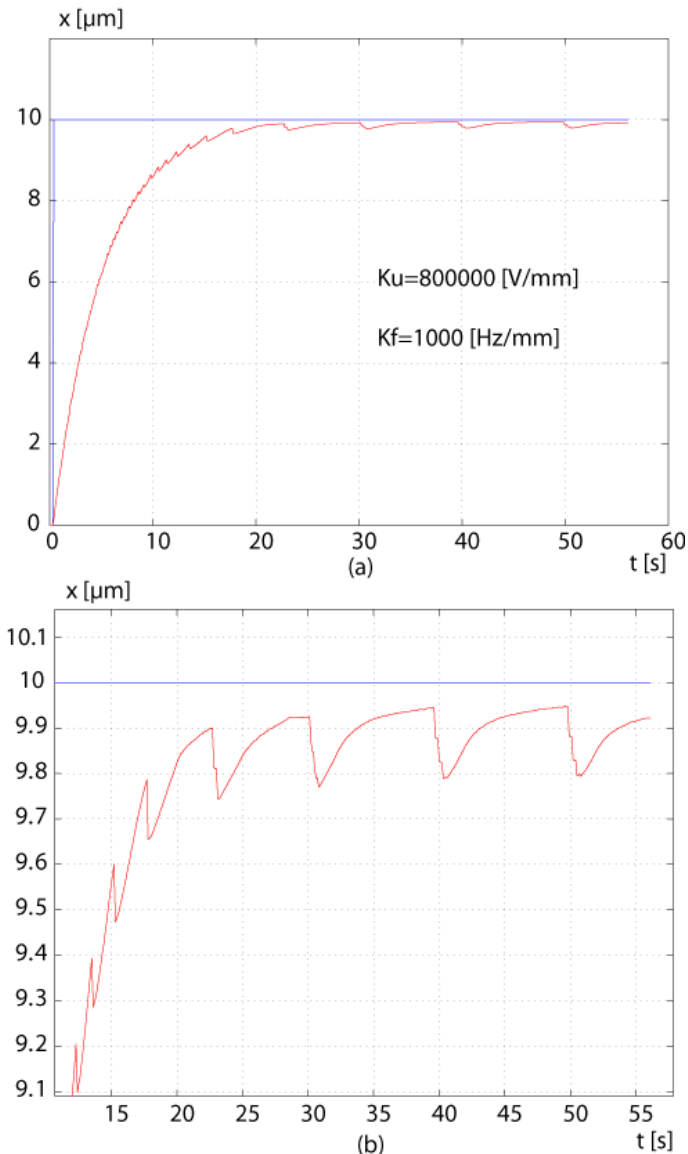

Fig. 15. U/f controller using an interferometer sensor. a: high stroke motion b: zoom of the final position.

\section{ACKNOWLEDGMENT}

Particular thanks to Dr Jean-Marc Breguet and team from the 'Laboratoire des Systèmes Robotiques' of the Swiss Federal Institute of Technology Lausanne (LSRO - EPFL) for accepting us to use their interferometer sensor. This work is partially supported by the EUPASS-project (www.eupass.org).

\section{REFERENCES}

[1] R.S. Fearing, "Survey of sticking effect for micro parts handling" IEEE International Conference on Intelligent Robots and Systems, IROS, pp212-227, Pittsburgh, PA, USA 1995.

[2] R. Matsuda and R. Kaneko, "Micro-step XY-stage using piezoelectric tube actuator" IEEE Micro Electro Mechanical Systems Conference, pp.137$142,1991$.

[3] S. Kleindiek and K. H. Herrmann, " A miniaturized scanning tunneling microscope with large operation range", Review of Scientific Instrument 64 (3), pp.692-693, March 1993.

[4] G. Mariotto and M. D'Angelo and I.V. Shvets, "Dynamic Behavior of a Piezowalker, Inertial and Frictional Configurations", Review of Scientific Instrument 70(9), pp.3651-3655, September 1999.

[5] T. Morita and R. Yoshida and Y. Okamoto and M. K. Kurosawa and T. Higuchi, "A Smooth Impact Rotation Motor Using a Multi-Layered Torsional Piezoelectric Actuator", IEEE Transactions on Ultrasonics, Ferroelectrics, and Frequency Control, 46(6), pp.1439-1445, November 1999.

[6] A. Bergander,W. Driesen, T. Varidel, M. Meizoso, and J.M. Breguet, "Mobile cm3-microbots with tools for nanoscale imaging and micromanipulation", Mechatronics and Robotics Conference, pp.1041-1047, 2004
[7] S. Fatikow, B. Magnussen, and U. Rembold, "A piezoelectric mobile robot for handling of microobjects", Microsystems, Intelligent Materials and Robots (MIMRS), pp.189-192, Sendai, Japan, September 1995.

[8] J.M. Breguet, R. Pérez, A. Bergander, C. Schmitt, R. Clavel and H. Bleuler, "Piezoactuators for motion control from centimeter to nanometer" IEEE International Conference on Intelligent Robots and Systems, IROS, pp-492-497, 2000

[9] A. Bergander and J.M. Breguet, "Performance improvements for stickslip positioners" Proceedings of 2003 IEEE International Symposium on Micromechatronics and Human Science (MHS), pp.59-66, October 2003.

[10] Babak Sedghi, "Control design of hybrid systems via dehybridization" PhD Thesis No2859, Ecole Polytechnique Fédérale de Lausanne, Switzerland 2003.

[11] J.M. Breguet and R. Clavel, "Stick and slip actuators: design, control, performances and applications" IEEE International Symposium on $\mathrm{Mi}$ cromechatronics and Human Science, pp.89-95, 1998.

[12] Micky Rakotondrabe, Yassine Haddab and Philippe Lutz, "Design, devbelopment and experiments of a high stroke-precision 2Dof (linearangular) microsystem" IEEE International Conference on Robotics and Automation, Orlando Florida, May 2006.

[13] M. Rakotondrabe, Y. Haddab and P. Lutz, "High-stroke motion modelling and voltage/frequency proportional control of a stick-slip microsystem" IEEE International Conference on Robotics and Automation, Roma Italy, April 2007. 